\title{
Leveraging the effects of chloroquine on resistant malaria parasites for combination therapies
}

\author{
Ana M. Untaroiu ${ }^{1,4 \dagger}$, Maureen A. Carey ${ }^{2,5 \dagger}$, Jennifer L. Guler ${ }^{3^{*}}$ and Jason A. Papin ${ }^{1 *}$ (D)
}

\begin{abstract}
Background: Malaria is a major global health problem, with the Plasmodium falciparum protozoan parasite causing the most severe form of the disease. Prevalence of drug-resistant $P$. falciparum highlights the need to understand the biology of resistance and to identify novel combination therapies that are effective against resistant parasites. Resistance has compromised the therapeutic use of many antimalarial drugs, including chloroquine, and limited our ability to treat malaria across the world. Fortunately, chloroquine resistance comes at a fitness cost to the parasite; this can be leveraged in developing combination therapies or to reinstate use of chloroquine.

Results: To understand biological changes induced by chloroquine treatment, we compared transcriptomics data from chloroquine-resistant parasites in the presence or absence of the drug. Using both linear models and a genome-scale metabolic network reconstruction of the parasite to interpret the expression data, we identified targetable pathways in resistant parasites. This study identified an increased importance of lipid synthesis, glutathione production/cycling, isoprenoids biosynthesis, and folate metabolism in response to chloroquine.

Conclusions: We identified potential drug targets for chloroquine combination therapies. Significantly, our analysis predicts that the combination of chloroquine and sulfadoxine-pyrimethamine or fosmidomycin may be more effective against chloroquine-resistant parasites than either drug alone; further studies will explore the use of these drugs as chloroquine resistance blockers. Additional metabolic weaknesses were found in glutathione generation and lipid synthesis during chloroquine treatment. These processes could be targeted with novel inhibitors to reduce parasite growth and reduce the burden of malaria infections. Thus, we identified metabolic weaknesses of chloroquine-resistant parasites and propose targeted chloroquine combination therapies.
\end{abstract}

Keywords: Malaria, Combination therapies, Chloroquine, Metabolic modeling

\section{Background}

There are approximately 3.2 billion people at risk of malaria infection worldwide and the malaria parasites cause half a million deaths annually [1]. Given the lack of a broadly effective vaccine, antimalarial drugs and protection from mosquito bites are essential in the control of malaria [2]. The most lethal species of the protozoan parasite that causes malaria, Plasmodium falciparum, has acquired resistance to every antimalarial drug on the

\footnotetext{
*Correspondence: jlg5fw@virginia.edu; papin@virginia.edu

${ }^{\dagger}$ Ana M. Untaroiu and Maureen A. Carey are co-first authors.

${ }^{3}$ Department of Biology, University of Virginia, Charlottesville, VA, USA

'Department of Biomedical Engineering, University of Virginia, Charlottesville, VA, USA

Full list of author information is available at the end of the article
}

market $[3,4]$. Since the development of novel antimalarials is slow, there is a need for combination therapies to target resistant parasites.

First introduced in 1934, chloroquine was a front-line antimalarial until the late 1950s when its heavy usage led to emergence of resistant $P$. falciparum strains near the Cambodia-Thailand border [5]. Chloroquine resistance has now been confirmed in over 40 countries, making resistance to this drug a global concern [5]. The mechanism of action of chloroquine is well studied. In intraerythrocytic trophozoite parasites, the drug blocks detoxification of heme, a byproduct of hemoglobin degradation [6]. During the asexual intraerythrocytic-stages, the parasite imports host cell hemoglobin into its food vacuole [7, 8]. Proteases in the food vacuole degrade hemoglobin into free amino

(c) The Author(s). 2019 Open Access This article is distributed under the terms of the Creative Commons Attribution 4.0 International License (http://creativecommons.org/licenses/by/4.0/), which permits unrestricted use, distribution, and 
acids which are utilized in various growth processes [9]. Heme is released during hemoglobin digestion and is essential for parasite growth as a cofactor for cytochromes in the parasite's electron transport chain $[10,11]$; however, elevated levels of intracellular heme can lead to cellular damage: oxidation of proteins, inhibition of proteases, and damage or lysis of membranes [12-14]. Heme released from hemoglobin is detoxified through three known mechanisms: [1] polymerization into hemozoin crystals, [2] detoxification through interactions with hydrogen peroxide in the food vacuole, and [3] a glutathione-mediated degradation process in the cytoplasm $[7,13,15,16]$. Chloroquine chemically binds to heme and the growing ends of hemozoin crystals, preventing crystallization-mediated heme detoxification $[17,18]$.

Chloroquine-resistant parasites are able to export chloroquine [19], which reduces the accessibility of chloroquine to its heme and hemozoin targets [20,21]. This resistance phenotype is mediated by mutations in the $P$. falciparum chloroquine resistance transporter ( $p f c r t$ ) gene that results in the removal of drug from its functional site, the food vacuole [22, 23]. Numerous mutations are associated with chloroquine resistance, depending on the genetic background, and result in varying degrees of resistance [22, 24-26]. However, the substitution of lysine to threonine at position 76 in pfcrt is found in all in vitro chloroquine-resistant parasites [27, 28].

Although chloroquine resistance is a well-studied case of antimalarial resistance, the mechanistic details and the broad phenotypic consequences of resistance remain poorly characterized. Studies on hemozoin formation estimate that only a third of heme released from hemoglobin is sequestered into hemozoin, suggesting a majority of heme is broken down through alternative, less well-characterized mechanisms, like peroxidative and glutathione degradation [16, 29]. Additional investigations are needed to understand the interplay between these mechanisms, which may become important in situations where resistant parasites are exposed to chloroquine. This situation is a greater possibility today, as some malaria endemic countries consider reintroducing chloroquine into their treatment regimens. Chloroquine resistance confers a fitness cost; thus, resistance alleles do not become fixed and decline in prevalence after chloroquine use is discontinued [30,31]. As a result, clinical trials have confirmed that reintroduction of chloroquine is highly efficacious $[32,33]$. Based on these findings, a reintroduction of chloroquine as a part of combination therapies may be an effective treatment strategy.

This study investigates the effects of chloroquine on chloroquine-resistant parasites to identify partner drugs that could be used in combination therapies to accelerate the reintroduction of chloroquine. We integrated the transcriptomics data from chloroquine-resistant parasites with or without chloroquine treatment [34] into a P. falciparum genome-scale metabolic network reconstruction to predict large-scale metabolic changes initiated by drug treatment, as we previously performed to study artemisinin resistance [35]. We identified shifts in metabolic flux and clinically available inhibitors that may partner well with chloroquine to target chloroquine resistant parasites.

\section{Methods \\ Differential gene analysis}

Expression data from in vitro $\mathrm{K} 1$ parasites untreated or treated with $\mathrm{EC}_{50}$ concentrations of chloroquine for $4 \mathrm{~h}$ and $24 \mathrm{~h}$ were used to investigate the transcriptional effects of chloroquine treatment (normalized expression data obtained via GEO accession number: GSE31109) [34]. Exposure for 4 and $24 \mathrm{~h}$ were defined as short and long-term treatment, respectively. Probes with missing expression values were set equal to the lowest expression value across all replicates for that probe [35]. Mean or median values across replicates were not used to replace missing expression values to avoid skewing the data with outliers. Genes with multiple probes were filtered from the data to ensure each gene corresponds to only one microarray probe. For repeating probes, only expression data from the probe with the largest variance across all replicates were retained. Means from repeating probes were not used to prevent averaging true signal with background noise. Probes with missing gene IDs were removed from the dataset.

The $\mathrm{R}$ package limma was used to conduct differential gene expression analysis (Fig. 1). Limma uses linear models to fit a data set and empirical Bayes statistical methods to determine variability and log-fold changes in gene expression [36]. Significance values from this analysis ( $p$-values) were modified using false discovery rate (FDR) correction in order to control for the parallel manner of comparing genes using limma, instead of testing genes in isolation. Differentially expressed genes were characterized as genes having a fold change (FC) greater than 2 or less than 0.5 and a FDR-adjusted $\mathrm{p}$-value less than 0.05 .

\section{Model curation}

Additional reactions were included into the $P$. falciparum model (iPfal17) based on supporting experimental evidence to generate our updated reconstruction [35]. More specifically, hydrogen peroxide was incorporated as a byproduct of hemoglobin digestion based on studies of the chemical steps in hemoglobin breakdown [37]. Reactions for heme degradation via glutathione and hydrogen peroxide were incorporated based on supporting literature $[38,39]$. See Additional file 1: Table S1 for modified reactions. 


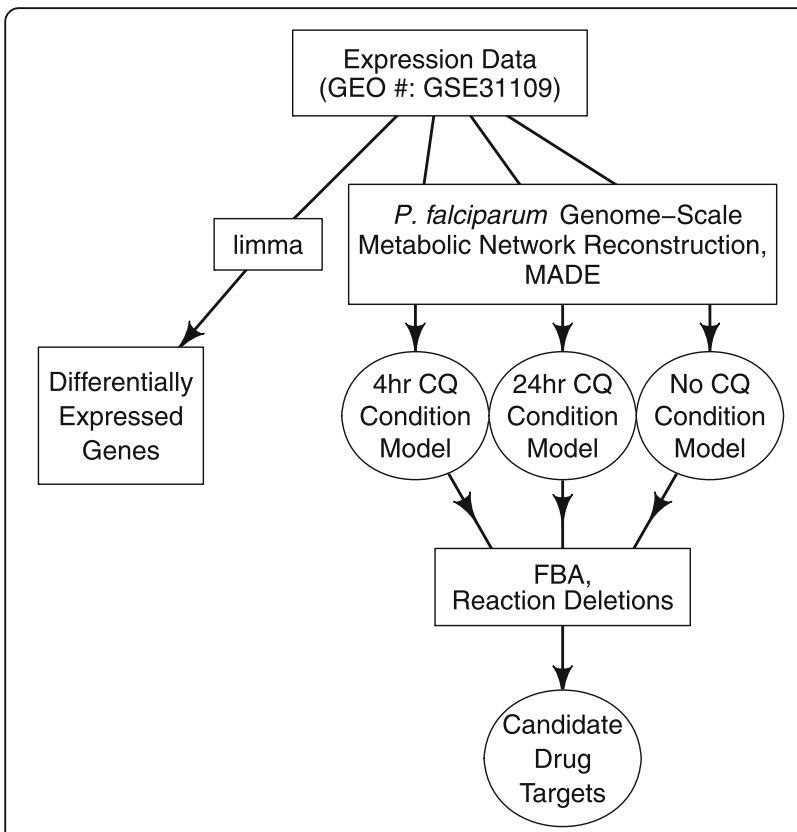

Fig. 1 Overview of Computational Approach. The R package limma was used to find differentially expressed genes (left square). Metabolic Adjustment for Differential Expression (MADE) algorithm was used to produce the condition-specific models (right 3 circles). Flux balance analysis (FBA) and simulated reaction deletions predicted drug targets for chloroquine-treated parasites. $C Q=$ Chloroquine

\section{Condition-specific model generation}

Condition-specific models were produced by integrating gene IDs with corresponding log FCs and FDR-adjusted $p$-values from the 4 and $24 \mathrm{~h}$ treatment conditions into a curated version (see above) of the intraerythrocytic-stage $P$. falciparum genome-scale metabolic network reconstruction [35] (Fig. 1). Data integration was conducted by using the Metabolic Adjustment for Differential Expression (MADE) algorithm; this algorithm accounts for the expression state of a gene with a weighted consideration of the statistical significance of the gene expression changes [40]. Gene expression data integration remains an open challenge in the metabolic modeling field and there are advantages and disadvantages to the methods that have emerged $[41,42]$. We selected MADE due to gene expression data availability and because this method does not require the user to select an arbitrary threshold to define significant or meaningful gene expression changes. Such thresholding can influence the resultant condition-specific model [41].

Condition-specific models were generated from a 'base' model, published in [35] with minor modifications documented in Additional file 1: Table S1. Models were constrained (using MADE) to maximize consistency between experimental expression data and presence of metabolic enzymes. Essentially, if a gene was expressed in a particular condition or essential for growth, the gene and its associated reactions were included the resultant condition-specific model. Condition-specific models were generated for each condition ( $4 \mathrm{~h}$ chloroquine-treated, 24 $\mathrm{h}$ chloroquine-treated, and untreated) using varying growth thresholds (30-80\%). This threshold represents the fraction of metabolic objective required for the output condition-specific model. We explored the effects of this threshold on condition-specific models to find minor differences in results. This paper reports common results across all models. It is important to emphasize that MADE integrates expression of all metabolic genes (not merely the DEGs identified through thresholding for significance and meaningful fold change) but prioritizes in silico consistency with in vitro observations based on statistical significance and fold change. In effect, MADE assumes larger, more significant changes in gene expression will have a larger effect on phenotype than smaller, less significant changes. However, MADE assumes small, less significant changes may still have an effect on phenotype. MADE also requires that the resultant condition-specific model is functional.

\section{Reaction essentiality predictions and flux analysis}

Essential reactions for each condition-specific model were generated by sequentially removing single reactions and testing the modified model for growth using flux balance analysis [43] (Fig. 1). Reactions were delineated as essential when the removal of the reaction from the model resulted in a decrease of the growth rate by $60 \%$ or more compared to the untreated model. This growth rate threshold was chosen to avoid a too conservative definition of essentiality, but to also ensure a significant growth defect. Existing algorithms in the COBRA toolbox were used to calculate ranges of possible flux levels through reactions in the condition-specific models [44, 45]. All simulations were performed in a nutrient rich environment permitting flux through all exchange, import, and export reactions, consistent with intraerythrocytic growth in vitro and in vivo. Generation of condition-specific models, reaction essentiality, and flux analysis were conducted using MATLAB vR2014a, COBRA Toolbox v3.0, and Gurobi 6.5.2 solver.

\section{Enrichment analysis}

The enrichment of metabolic subsystems among essential reactions was investigated. The purpose of this analysis is to determine whether a certain subsystem appears to a greater or lesser extent than expected by chance. Corresponding metabolic subsystems for genes and reactions were derived from the genome-scale metabolic model used in this study [35]. We tested for enrichment of subsystems among essential genes with reference to the unconstrained model using a Fisher's exact test. Significant subsystems were defined as having a FDR-adjusted $p$-value 
less than 0.05. RStudio v3.3.0 was used for the differential gene and enrichment analysis.

\section{Results}

Resistant parasites respond to chloroquine pressure with moderate but statistically significant gene downregulation

Since this analysis was not performed in the original study [34], differentially expressed genes (DEGs) were identified for both short and long-term treatment relative to untreated trophozoite expression. The majority of genes do not show a significant change in expression in response to chloroquine treatment (genes represented by points in black, Fig. 2). Out of a total of 5121 genes, there were 97 and 166 differentially expressed genes (DEGs) in short- and long-term treatment conditions, respectively (represented by points in red, Fig. 2). In both conditions, more genes were significantly down-regulated than up-regulated in response to chloroquine treatment $(95.9 \%$ for short-term and 83.1\% for long-term treatment, fold change less than 0.5, Additional file 1: Table S2). Up-regulated genes showed very moderate over-expression (fold change between 2 and 2.5) and included one metabolic gene (out of 482 metabolic genes, Additional file 1: Table S3). No evidence was found in the literature to suggest these DEGs are directly involved in chloroquine treatment or resistance.

\section{Identifying metabolic weakness of chloroquine-treated resistant parasites using metabolic modeling}

In order to understand the system-wide context for these gene expression changes, we integrated transcriptomics data into a genome-scale metabolic network reconstruction of intraerythrocytic-stage $P$. falciparum to generate three condition-specific models (untreated, short- and long-term chloroquine treatment). We then predicted reactions essential for parasite growth. One hundred and sixty-four metabolic reactions (out of 1197) are essential in all three models (Fig. 3a, center); these reactions represent core metabolic pathways of the parasite.
Reactions that become essential during chloroquine treatment highlight weak points in the metabolic network and can be exploited as drug targets in chloroquine-resistant parasites. We found 210 and 208 essential metabolic reactions in short- and long-term treatment, respectively. Thirty-two were shared between the two conditions (Additional file 1: Table S5, Fig. 3); fifteen of these are involved in phosphatidylethanolamine and phosphatidylserine metabolism, seven in phospholipid utilization, and one in the import of reduced glutathione (examples summarized in Fig. 3b; Additional file 1: Table S5). When we performed subsystem enrichment on the essential reactions, the following subsystems were over-represented: phosphatidylethanolamine and phosphatidylserine metabolism ( $p$-value $=$ $4.84 \mathrm{E}-6)$, tRNA synthesis $(p$-value $=0.024)$, and phospholipid utilization $(p$-value $=0.037$ ). Reactions involved in transport and lipid metabolism were under-represented in chloroquine treated models $(p$-value $=0.0061$ and $2.51 \mathrm{E}-8$, respectively).

Fourteen reactions are essential in only the short-term treatment model (Fig. 3a in blue, Additional file 1: Table S6). Of these essential reactions, five reactions are involved in the de novo synthesis of thiamine diphosphate, the active form of vitamin B1 (Fig. 4 in white). Eight reactions were uniquely essential in the long-term treatment model (Fig. 3a in green, Additional file 1: Table S7), notably the conversion of chorismate into 4-aminobenzoate for folate metabolism is essential (Fig. 5 in black).

We explored why thiamine diphosphate synthesis was essential only in the short-term treatment model. $P$. falciparum has an alternate route for thiamine diphosphate production (termed 'import pathway', Fig. 4), where thiamine is imported into the parasite's cytoplasm and phosphorylated by thiamine diphosphokinase to form thiamine diphosphate. For the untreated and long-term treatment models, only the import pathway is active. For the short-term treatment model, only the de novo synthesis pathway has activity. These results suggest a switch in thiamine diphosphate production in early stages of drug treatment.
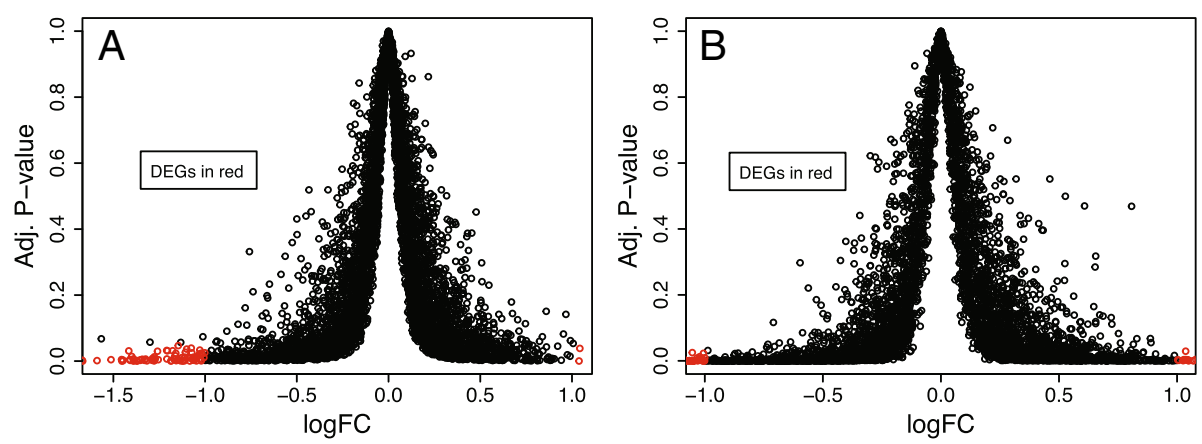

Fig. 2 Resistant Parasites Respond to Drug by Downregulating Expression. FDR-adjusted $p$-values and fold changes (FC, reported as log values) for genes during (a) short-term treatment and (b) long-term treatment with chloroquine. Adjusted p-values represent the significance level of changes in expression. Fold change quantifies the variation in the gene expression relative to untreated resistant parasite expression. DEGs = differentially expressed genes 

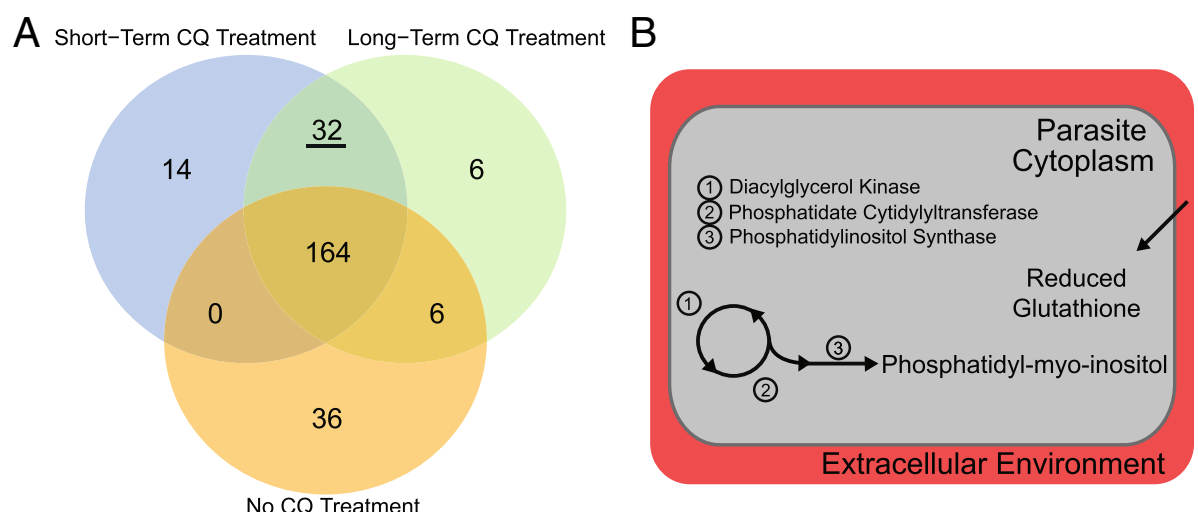

Fig. 3 Chloroquine-treated parasites have new metabolic weaknesses. a Comparison of essential reactions in the three condition-specific models (one for each condition: short-term and long-term chloroquine treatment, as well as the untreated condition). Summarized, 7\% of essential reactions during shortterm treatment are unique to that condition, 3\% of essential reactions during long-term treatment are unique to that condition, and 17\% of essential reactions in the untreated condition are unique to that condition. Lastly, 12\% of all essential reactions are shared by both treatment conditions. Note: 159 reactions are essential prior to constraining the model to represent condition-specific metabolism. $\mathbf{b}$ lllustration of common essentiality predictions (underlined in A) between the drug-treatment models, including inositol phosphate metabolism and glutathione import are represented. These enzymes could be targeted in these resistant parasites in combination with chloroquine; resultant combination therapies would specifically target resistant parasites during chloroquine treatment, not wild-type parasites or single-drug treatment. Red region depicts the host red blood cell and grey is the parasite's cytoplasm

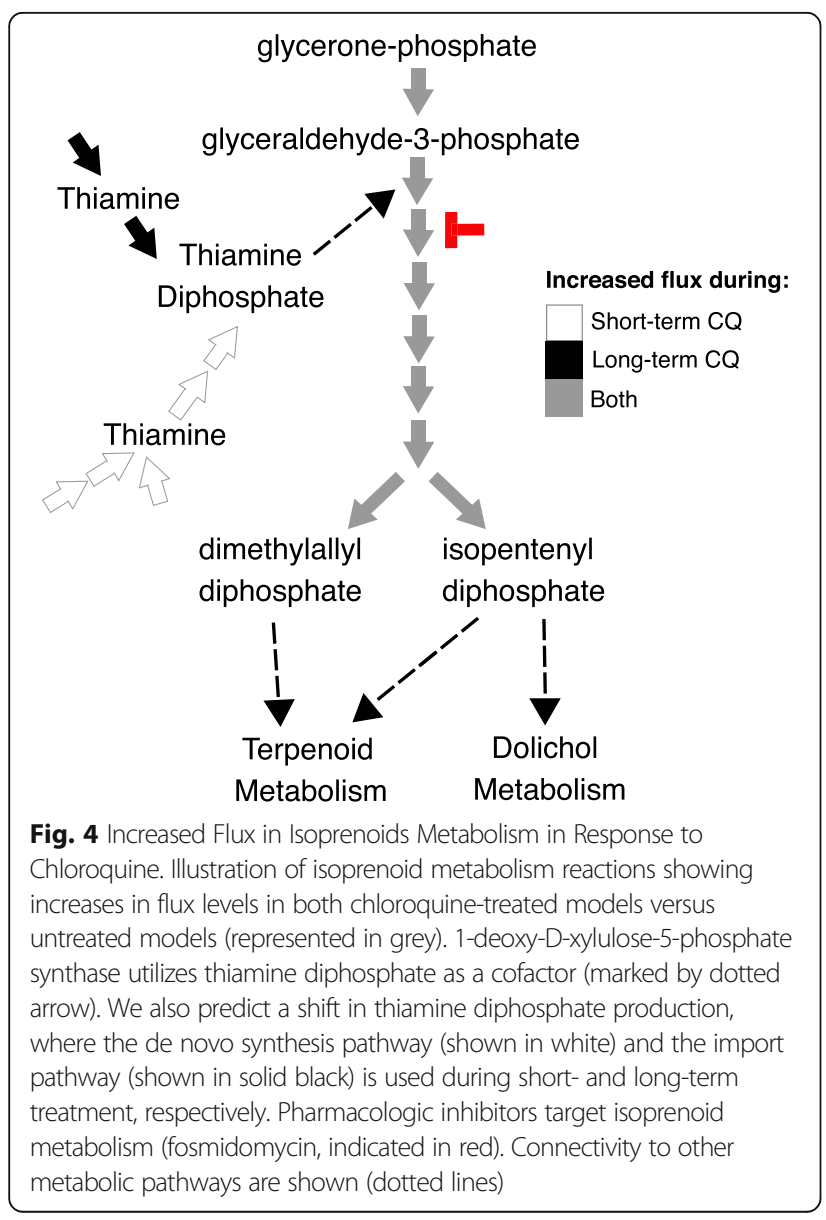

Six enzymes are known to utilize thiamine diphosphate (also called thiamine pyrophosphate) as a cofactor in $P$. falciparum [46-50]. Flux levels of these reactions predicted from flux balance analysis were investigated to understand thiamine diphosphate usage and essentiality. Flux balance analysis simulates steady-state flux values through the network's reactions and predicts the reactions needed to maximize the objective function, which was selected as the biomass equation; thus, this analysis predicts reactions needed for growth. Four of these thiamine diphosphate dependent enzymes (pyruvate dehydrogenase, 2-oxoglutarate dehydrogenase, 3-methyl-2-oxobutanoate dehydrogenase, and branched-chain-alpha-keto-acid dehydrogenase) are predicted to carry no flux in treated and untreated parasites. In response to drug treatment, flux changes through two thiamine diphosphate-dependent reactions: flux through the reaction catalyzed by 1-deoxy-Dxylulose phosphate synthase increases (in isoprenoids metabolism) and flux through the reaction catalyzed by transketolase decreases (in pentose phosphate pathway). Flux of the other reactions in isoprenoids metabolism are also consistently greater in response to chloroquine treatment (Fig. 4 in grey; Additional file 1: Table S8). Compared to untreated models, short- and long-term treatment show a $62-83$ and $24 \%$ increase in flux, respectively.

To next focus on the reactions essential in only the long-term treatment model, we explored the utilization of chorismate, a precursor of folate. During long-term chloroquine treatment, synthesis of 4-aminobenzoate from chorismate is used, rather than importing 4-aminobenzoate from the host cell (Fig. 5). Thus, the 


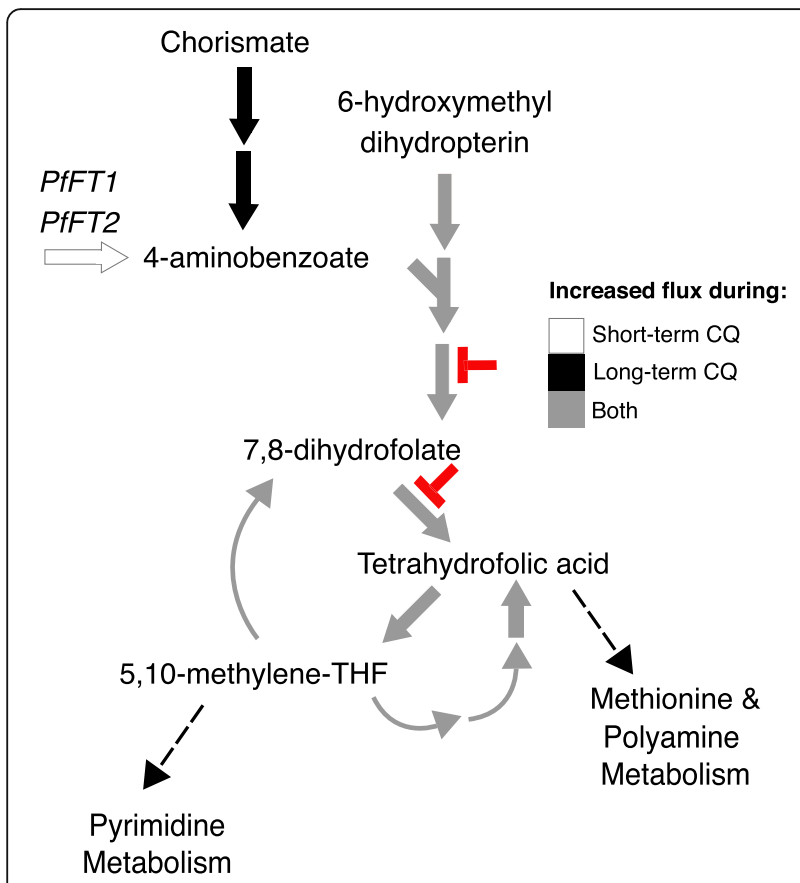

Fig. 5 Increased Flux in Folate Metabolism in Response to Chloroquine. Illustration of folate metabolism reactions showing increases in flux levels in both chloroquine-treated models versus untreated models. We predict a shift in 4-aminobenzoate acquisition between short- and long-term chloroquine treatment, and elevated flux through all downstream steps of folate metabolism. Clinically available drugs target this pathway (sulfadoxine and pyrimethamine, indicated in red). Both enzymes targeted by these antimalarials (dihydropteroate synthase and dihydrofolate reductase) carry increased flux in both models of short- and long-term chloroquine treatment. Connectivity to other metabolic pathways are shown (dotted lines)

conversion of chorismate into 4-aminobenzoate is essential. In contrast, short-term chloroquine-treated parasites predominately rely on membrane folate transporters (PfFT1 and PfFT2) for 4-aminobenzoate (Fig. 5). Downstream steps in folate metabolism, including dihydropteroate synthase and dihydrofolate reductase, carry a 62.4 and $24.2 \%$ increase in flux during short- and long-term chloroquine treatment, respectively (Fig. 5 in grey; Additional file 1: Table S9).

\section{Discussion}

In this study, we used transcriptomics data to investigate metabolic shifts resulting from chloroquine treatment of resistant parasites. We performed differential expression analysis and integrated expression data into a genomescale metabolic model to identify targetable weaknesses of chloroquine-treated resistant parasites. Using this approach, we identified metabolic pathways that, if targeted, could be developed as partner drugs for chloroquine combination therapies.

\section{Chloroquine affects resistant parasites}

Importantly, we observed a significant down-regulation of genes in response to chloroquine treatment (Fig. 2). This suggests that chloroquine treatment continues to affect the resistant parasite, despite the parasite's resistance to the drug's cytocidal effects. This observation is consistent with growth defects observed in chloroquinetreated resistant parasites (20 and $45 \%$ inhibition after 8 and $48 \mathrm{~h}$, respectively [51]). Moreover, the effect of chloroquine is not on a single gene and the parasite's response is not just a function of a single gene, as evident by the numerous differentially expressed genes and the multiple nonspecific resistance alleles [52, 53]. Longer exposure time exacerbates this effect, as illustrated by the increase in DEGs with the 24-h drug treatment (Fig. 2). Moreover, the parasite's response to chloroquine is not static, as there are DEGs and essential genes associated with short-term treatment but not long-term treatment.

\section{Novel proposed targets against chloroquine-resistant parasites}

We propose that resistant parasites are using different metabolic pathways when in the presence of chloroquine because there are treatment-associated essential reactions. Subsystem enrichment of essentiality predictions suggests many shifts in lipid metabolism. The increased importance of phospholipids in both treatment conditions (Fig. 3b) may represent the parasite's attempt to counteract the effects of chloroquine treatment. This response could occur through a number of routes: first, additional lipid species may be required to repair cellular membranes damaged by the build-up of intracellular heme during chloroquine treatment [14]. Second, lipids themselves have been shown to contribute to the detoxification of heme into hemozoin $[54,55]$. The demand for lipids for these roles may represent a targetable metabolic weakness of drug-treated chloroquine-resistant parasites.

The transport of glutathione from the extracellular environment into the cytoplasm is predicted to be essential during chloroquine treatment (Fig. 3b). Glutathione is involved in the degradation of non-polymerized heme, in addition to being involved in managing oxidative stress in the parasite [15]. Since these reactions are only essential during chloroquine treatment, this result suggests the activation of these reactions may be a direct result of drug pressure placed on the parasite and the accompanying cellular damage. This result is supported by observed correlations between chloroquine resistance and intracellular glutathione levels [56-58]. The competitive inhibition of glutathione degradation by heme also supports the increased importance of glutathione accumulations to counteract chloroquine pressures [59]. Thus, glutathione is essential in combating the effects of 
chloroquine and can be considered another metabolic weakness of resistant parasites.

\section{Partner drugs for chloroquine combination therapies}

Folate metabolism is needed for DNA synthesis and metabolism of certain amino acids [54]. Interestingly, downstream steps in folate metabolism, including dihydropteroate synthase and dihydrofolate reductase, are predicted to carry more flux during chloroquine treatment (Fig. 5), implying they are necessary for survival or tend to be overexpressed during treatment. This result suggests that this pathway has increased importance under chloroquine treatment and could be targeted in combination therapies. Recent clinical use of such a combination therapy supports this conclusion; chloroquine in combination with inhibitors of dihydrofolate reductase and dihydropteroate synthase (sulfadoxine-pyrimethamine) is effective against chloroquine-resistant parasites [60-62]. Our results suggest that chloroquine-resistant parasites are more susceptible to these drugs than sensitive parasites and our modeling approach provides a mechanistic explanation for this drug synergy.

Unique to our study, we predict a novel role for isoprenoids synthesis in chloroquine-resistant parasites. Under chloroquine treatment, there is increased flux through reactions in the non-mevalonate pathway for isoprenoids metabolism (Fig. 4), the only synthesis pathway for isoprenoids in P. falciparum [63, 64]. This pathway is thiamine diphosphate-dependent and we also observed a switch in thiamine scavenging to de novo synthesis (Fig. 4), highlighting the dynamic state of these pathways. Our computational analysis suggests that chloroquine-resistant parasites have increased susceptibility to non-mevalonate pathway inhibitors, such as fosmidomycin and its derivative, FR-900098 (Fig. 4) [65]. This metabolic weakness represents an ideal target since the non-mevalonate pathway is constitutively essential, with increased usage in chloroquine-treated resistant parasites [66]. Fosmidomycin alone is moderately effective against chloroquine-resistant parasites [67] and there is no additivity between fosmidomycin and chloroquine in vitro in a screen of pooled chloroquine-sensitive and resistant parasites [68]. We hypothesize that in a population of chloroquine resistant parasites, there would be additivity between fosmidomycin and chloroquine; thus, we expect that these parasites would be even more susceptible to fosmidomycin while under chloroquine treatment both in vitro and in vivo.

Folates play an important role in DNA synthesis (through pyrimidine synthesis), protection from DNA damage (though DNA-stabilizing polyamines), and DNA methylation (also through polyamine methyl donors), and upregulated folate metabolism may be indicative of the cell's need to repair DNA damage. We predict resistant parasites are well prepared to synthesize DNA and respond to DNA damage by elevating their folate pool. Previous studies find that chloroquine inhibits DNA repair in mammalian cells [69], and thus, resistant parasites may be able to better tolerate the off-target effects of chloroquine on DNA by increasing their folate pool. Thus, we have proposed a novel combination therapy involving readily available antimalarials that may inhibit the growth of chloroquine-resistant parasites by targeting the noncanonical effects of chloroquine.

\section{Conclusions}

The fitness cost associated with chloroquine resistance means that once drug pressure is removed, sensitive parasites again become prominent in the population. Due to its low cost and easy access in malaria endemic countries, chloroquine use is being considered in areas where the return to sensitivity has been confirmed. However, we must be deliberate about how this drug is reinstated to avoid the rapid return of resistant parasites. Thus, we identified potential drug targets for chloroquine combination therapies, using metabolic modeling of resistant parasites under chloroquine treatment. Significantly, we predict that the combination of chloroquine with sulfadoxine-pyrimethamine or fosmidomycin may be more effective against chloroquine-resistant parasites than either drug alone; further studies will explore the use of these drugs as chloroquine resistance blockers. Additional metabolic weaknesses were found in glutathione generation and lipid synthesis during chloroquine treatment. These processes could be targeted with novel inhibitors to reduce parasite growth, thus reducing the burden of malaria infections.

\section{Additional file}

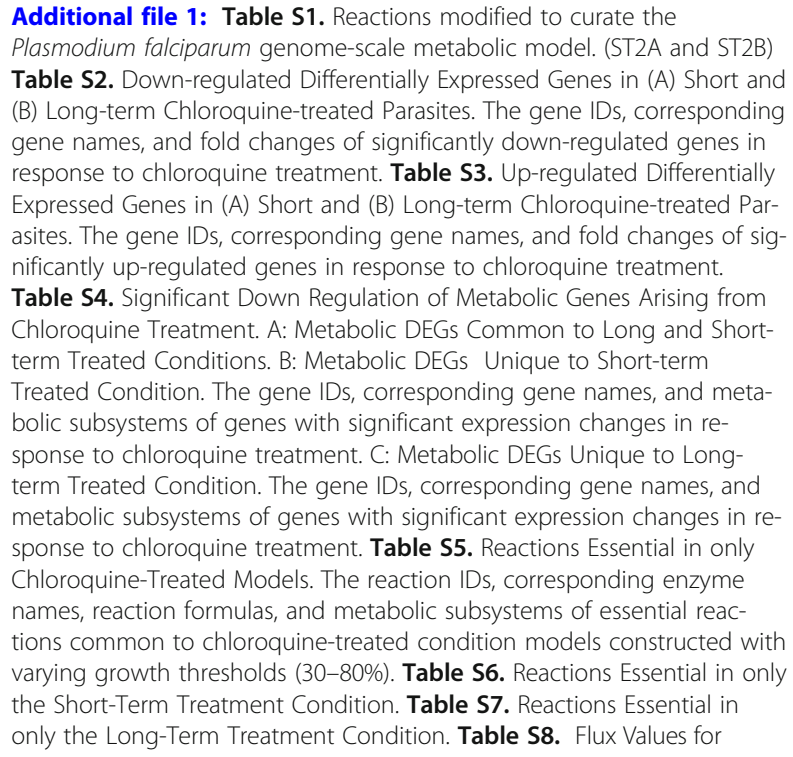


Isoprenoid Metabolism Reactions. Results represent median flux values across condition models constructed with varying growth thresholds (3080\%). Bolded reactions are represented in Fig. 4. Table S9. Flux Values for Folate Metabolism Reactions. Results represent median flux values across condition models constructed with varying growth thresholds (30-80\%). Bolded reactions are represented in Fig. 5. (ODS 28 kb)

\section{Abbreviations}

CQ: Chloroquine; DEG: Differentially expressed gene; FBA: Flux balance analysis; FC: Fold change; FDR: False-discovery rate; MADE: Metabolic Adjustment for Differential Expression

\section{Acknowledgements}

We thank the members of the Guler and Papin labs for their thoughtfu feedback and the openCobra community for software development integral to this work.

\section{Funding}

The study was financed by the National Institute of Allergy and Infectious Disease (R21Al119881 - JG and JP), an institutional training grant (T32GM008136 - MC), and the Arnold and Mabel Beckman Foundation (via the Beckman Scholars program - AU). No funding agency participated in the study design, data collection, data analysis, interpretation of data, or writing of the manuscript.

\section{Availability of data and materials}

The publicly available dataset analyzed during the current study are available on the NCBI's Gene Expression Omnibus, GSE31109. Additionally, our curated model and code are available on GitHub, at https://github.com/csbl/ Chloroquine-Project.

\section{Authors' contributions}

$A U$ and $M C$ designed the study. AU curated the model and performed statistical and network analyses. $\mathrm{AU}$ and $\mathrm{MC}$ interpreted the data and analyses. AU wrote the manuscript. MC, JG, and JP edited the manuscript. JG and JP provided resources. All authors read and approved the final manuscript.

\section{Ethics approval and consent to participate}

Not applicable.

\section{Consent for publication}

Not applicable.

\section{Competing interests}

The authors declare that they have no competing interests.

\section{Publisher's Note}

Springer Nature remains neutral with regard to jurisdictional claims in published maps and institutional affiliations.

\section{Author details}

'Department of Biomedical Engineering, University of Virginia, Charlottesville, VA, USA. 'Department of Microbiology, Immunology, and Cancer Biology, University of Virginia, Charlottesville, VA, USA. ${ }^{3}$ Department of Biology, University of Virginia, Charlottesville, VA, USA. ${ }^{4}$ Present address: Department of Radiology and Medical Imaging, University of Virginia, Charlottesville, VA, USA. ${ }^{5}$ Present address: Division of Infectious Diseases and International Health, Department of Medicine, University of Virginia, Charlottesville, VA, USA.

\section{Received: 11 March 2019 Accepted: 19 March 2019}

\section{Published online: 15 April 2019}

\section{References}

1. WHO. World Malaria Report 2015: WHO. Available from: http://www.who.int/ malaria/publications/world-malaria-report-2015/report/en/. [cited 12 Apr 2017 ]

2. Russell PK, Howson CP. Vaccines against malaria: Hope in a gathering storm [internet]: National Academies Press (US); 1996. Available from: https://www. ncbi.nlm.nih.gov/books/NBK231030/. [cited 18 Apr 2017]
3. Chakraborty A. Emerging drug resistance in Plasmodium falciparum: a review of well-characterized drug targets for novel antimalarial chemotherapy. Asian Pac J Trop Dis. 2016;6(7):581-8.

4. Cowman AF, Healer J, Marapana D, Marsh K. Malaria: biology and disease. Cell. 2016;167(3):610-24.

5. Payne D. Spread of chloroquine resistance in Plasmodium falciparum. Parasitol Today Pers Ed. 1987;3(8):241-6

6. Chou AC, Chevli R, Fitch CD. Ferriprotoporphyrin IX fulfills the criteria for identification as the chloroquine receptor of malaria parasites. Biochemistry. 1980;19(8):1543-9.

7. Francis SE, Sullivan DJ, Goldberg DE. Hemoglobin metabolism in the malaria parasite Plasmodium falciparum. Annu Rev Microbiol. 1997;51:97-123.

8. Goldberg DE, Slater AF, Cerami A, Henderson GB. Hemoglobin degradation in the malaria parasite Plasmodium falciparum: an ordered process in a unique organelle. Proc Natl Acad Sci U S A. 1990;87(8):2931-5.

9. Goldberg DE, Slater AF, Beavis R, Chait B, Cerami A, Henderson GB. Hemoglobin degradation in the human malaria pathogen Plasmodium falciparum: a catabolic pathway initiated by a specific aspartic protease. J Exp Med. 1991;173(4):961-9.

10. Fry M, Pudney M. Site of action of the antimalarial hydroxynaphthoquinone, 2-[trans-4-(4'-chlorophenyl) cyclohexyl]-3- hydroxy-1,4-naphthoquinone (566C80). Biochem Pharmacol. 1992;43(7):1545-53.

11. Srivastava IK, Rottenberg $H$, Vaidya AB. Atovaquone, a broad Spectrum Antiparasitic drug, collapses mitochondrial membrane potential in a malarial parasite. J Biol Chem. 1997;272(7):3961-6.

12. Francis SE, Gluzman IY, Oksman A, Knickerbocker A, Mueller R, Bryant ML, et al. Molecular characterization and inhibition of a Plasmodium falciparum aspartic hemoglobinase. EMBO J. 1994;13(2):306-17.

13. Sigala PA, Goldberg DE. The peculiarities and paradoxes of Plasmodium heme metabolism. Annu Rev Microbiol. 2014:68:259-78.

14. Vander Jagt DL, Hunsaker LA, Campos NM. Characterization of a hemoglobin-degrading, low molecular weight protease from Plasmodium falciparum. Mol Biochem Parasitol. 1986;18(3):389-400.

15. Zhang J, Krugliak M, Ginsburg H. The fate of ferriprotorphyrin IX in malaria infected erythrocytes in conjunction with the mode of action of antimalarial drugs. Mol Biochem Parasitol. 1999:99(1):129-41.

16. Loria P, Miller S, Foley M, Tilley L. Inhibition of the peroxidative degradation of haem as the basis of action of chloroquine and other quinoline antimalarials. Biochem J. 1999;339(Pt 2):363-70.

17. Sullivan DJ, Gluzman IY, Russell DG, Goldberg DE. On the molecular mechanism of chloroquine's antimalarial action. Proc Natl Acad Sci U S A. 1996;93(21):11865-70.

18. Pagola S, Stephens PW, Bohle DS, Kosar AD, Madsen SK. The structure of malaria pigment beta-haematin. Nature. 2000;404(6775):307-10.

19. Fitch CD. Plasmodium falciparum in owl monkeys: drug resistance and chloroquine binding capacity. Science. 1970;169(3942):289-90.

20. Sanchez CP, McLean JE, Rohrbach P, Fidock DA, Stein WD, Lanzer M. Evidence for a pfcrt-associated chloroquine efflux system in the human malarial parasite Plasmodium falciparum. Biochemistry, 2005:44(29):9862-70.

21. Krogstad DJ, Gluzman IY, Kyle DE, Oduola AM, Martin SK, Milhous WK, et al. Efflux of chloroquine from Plasmodium falciparum: mechanism of chloroquine resistance. Science. 1987;238(4831):1283-5.

22. Fidock DA, Nomura T, Talley AK, Cooper RA, Dzekunov SM, Ferdig MT, et al. Mutations in the P. Falciparum digestive vacuole transmembrane protein PfCRT and evidence for their role in chloroquine resistance. Mol Cell. 2000:6(4):861-71.

23. Roepe PD. PfCRT-mediated drug transport in malarial parasites. Biochemistry. 2011:50(2):163-71.

24. Martin SK, Oduola AM, Milhous WK. Reversal of chloroquine resistance in Plasmodium falciparum by verapamil. Science. 1987;235(4791):899-901.

25. Foote SJ, Thompson JK, Cowman AF, Kemp DJ. Amplification of the multidrug resistance gene in some chloroquine-resistant isolates of $\mathrm{P}$. Falciparum. Cell. 1989:57(6):921-30.

26. Wilson CM, Serrano AE, Wasley A, Bogenschutz MP, Shankar AH, Wirth DF. Amplification of a gene related to mammalian mdr genes in drug-resistant Plasmodium falciparum. Science. 1989;244(4909):1184-6.

27. Djimdé A, Doumbo OK, Cortese JF, Kayentao K, Doumbo S, Diourté Y, et al. A molecular marker for chloroquine-resistant falciparum malaria. N Engl J Med. 2001;344(4):257-63.

28. Wootton JC, Feng X, Ferdig MT, Cooper RA, Mu J, Baruch DI, et al. Genetic diversity and chloroquine selective sweeps in Plasmodium falciparum. Nature. 2002:418(6895):320-3. 
29. Nagababu E, Chrest FJ, Rifkind JM. Hydrogen-peroxide-induced heme degradation in red blood cells: the protective roles of catalase and glutathione peroxidase. Biochim Biophys Acta. 2003;1620(1):211-7.

30. Kublin JG, Cortese JF, Njunju EM, Mukadam RAG, Wirima JJ, Kazembe PN, et al. Reemergence of chloroquine-sensitive Plasmodium falciparum malaria after cessation of chloroquine use in Malawi. J Infect Dis. 2003;187(12):1870-5.

31. Mita T, Kaneko A, Lum JK, Bwijo B, Takechi M, Zungu IL, et al. Recovery of chloroquine sensitivity and low prevalence of the Plasmodium falciparum chloroquine resistance transporter gene mutation K76T following the discontinuance of chloroquine use in Malawi. Am J Trop Med Hyg. 2003; 68(4):413-5

32. Laufer MK, Thesing PC, Eddington ND, Masonga R, Dzinjalamala FK, Takala SL, et al. Return of chloroquine antimalarial efficacy in Malawi. N Engl J Med. 2006; 355(19):1959-66.

33. Laufer MK, Thesing PC, Dzinjalamala FK, Nyirenda OM, Masonga R, Laurens $\mathrm{MB}$, et al. A longitudinal trial comparing chloroquine as monotherapy or in combination with Artesunate, azithromycin or Atovaquone-Proguanil to treat malaria. PLoS One. 2012;7(8):e42284.

34. Kritsiriwuthinan K, Chaotheing S, Shaw PJ, Wongsombat C, ChavalitshewinkoonPetmitr P, Kamchonwongpaisan S. Global gene expression profiling of Plasmodium falciparum in response to the anti-malarial drug pyronaridine. Malar J. 2011;10:242.

35. Carey MA, Papin JA, Guler JL. Novel Plasmodium falciparum metabolic network reconstruction identifies shifts associated with clinical antimalarial resistance. BMC Genomics. 2017;18(1):543.

36. Ritchie ME, Phipson B, Wu D, Hu Y, Law CW, Shi W, et al. Limma powers differential expression analyses for RNA-sequencing and microarray studies. Nucleic Acids Res. 2015;43(7):e47.

37. Wallace WJ, Houtchens RA, Maxwell JC, Caughey WS. Mechanism of autooxidation for hemoglobins and myoglobins. Promotion of superoxide production by protons and anions. J Biol Chem. 1982;257(9):4966-77.

38. Atamna $\mathrm{H}$, Ginsburg $\mathrm{H}$. Heme degradation in the presence of glutathione a proposed mechanism to account for the high levels of non-heme iron found in the membranes of hemoglobinopathic red blood cells. J Biol Chem. 1995;270(42):24876-83.

39. Brown SB, Hatzikonstantinou H, Herries DG. The role of peroxide in haem degradation. A study of the oxidation of ferrihaems by hydrogen peroxide. Biochem J. 1978;174(3):901-7.

40. Jensen PA, Papin JA. Functional integration of a metabolic network model and expression data without arbitrary thresholding. Bioinformatics. 2011; 27(4):541-7.

41. Opdam S, Richelle A, Kellman B, Li S, Zielinski DC, Lewis NE. A systematic evaluation of methods for tailoring genome-scale metabolic models. Cell Syst. 2017:4(3):318-329.e6.

42. Machado D, Herrgård M. Systematic evaluation of methods for integration of transcriptomic data into constraint-based models of metabolism. PLoS Comput Biol. 2014;10(4):e1003580.

43. Orth JD, Thiele I, Palsson B $\varnothing$. What is flux balance analysis? Nat Biotechnol. 2010;28(3):245-8.

44. Gudmundsson S, Thiele I. Computationally efficient flux variability analysis. BMC Bioinf. 2010;11:489.

45. Becker SA, Feist AM, Mo ML, Hannum G, Palsson B $\varnothing$, Herrgard MJ. Quantitative prediction of cellular metabolism with constraint-based models: the COBRA toolbox. Nat Protoc. 2007;2(3):727-38.

46. Foth BJ, Stimmler LM, Handman E, Crabb BS, Hodder AN, GI MF. The malaria parasite Plasmodium falciparum has only one pyruvate dehydrogenase complex, which is located in the apicoplast. Mol Microbiol. 2005;55(1):39-53.

47. Oppenheim RD, Creek DJ, Macrae Jl, Modrzynska KK, Pino P, Limenitakis J, et al. $\mathrm{BCKDH}$ : the missing link in apicomplexan mitochondrial metabolism is required for full virulence of toxoplasma gondii and Plasmodium berghei. PLoS Pathog. 2014;10(7):e1004263.

48. McMillan PJ, Stimmler LM, Foth BJ, McFadden Gl, Müller S. The human malaria parasite Plasmodium falciparum possesses two distinct dihydrolipoamide dehydrogenases. Mol Microbiol. 2005;55(1):27-38.

49. Goswami AM. Computational analysis, structural modeling and ligand binding site prediction of Plasmodium falciparum 1-deoxy-d-xylulose-5phosphate synthase. Comput Biol Chem. 2017;66:1-10.

50. Joshi S, Singh AR, Kumar A, Misra PC, Siddiqi MI, Saxena JK. Molecular cloning and characterization of Plasmodium falciparum transketolase. Mol Biochem Parasitol. 2008;160(1):32-41.
51. Yayon A, Vande Waa JA, Yayon M, Geary TG, Jensen JB. Stage-dependent effects of chloroquine on Plasmodium falciparum in vitro. J Protozool. 1983;30(4):642-7.

52. Foote SJ, Kyle DE, Martin RK, Oduola AMJ, Forsyth K, Kemp DJ, et al. Several alleles of the multidrug-resistance gene are closely linked to chloroquine resistance in Plasmodium falciparum. Nature. 1990;345(6272):255-8.

53. Sidhu ABS, Verdier-Pinard D, Fidock DA. Chloroquine resistance in Plasmodium falciparum malaria parasites conferred by pfcrt mutations. Science. 2002;298(5591):210-3.

54. Egan TJ. Haemozoin formation. Mol Biochem Parasitol. 2008;157(2):127-36.

55. Pisciotta JM, Coppens I, Tripathi AK, Scholl PF, Shuman J, Bajad S, et al. The role of neutral lipid nanospheres in Plasmodium falciparum haem crystallization. Biochem J. 2007:402(Pt 1):197-204.

56. Dubois VL, Platel DF, Pauly G, Tribouley-Duret J. Plasmodium berghei: implication of intracellular glutathione and its related enzyme in chloroquine resistance in vivo. Exp Parasitol. 1995;81(1):117-24.

57. Platel DF, Mangou F, Tribouley-Duret J. Role of glutathione in the detoxification of ferriprotoporphyrin IX in chloroquine resistant Plasmodium berghei. Mol Biochem Parasitol. 1999;98(2):215-23.

58. Meierjohann S, Walter RD, Müller S. Regulation of intracellular glutathione levels in erythrocytes infected with chloroquine-sensitive and chloroquineresistant Plasmodium falciparum. Biochem J. 2002;368(3):761-8.

59. Famin $\mathrm{O}$, Krugliak $\mathrm{M}$, Ginsburg $\mathrm{H}$. Kinetics of inhibition of glutathionemediated degradation of ferriprotoporphyrin IX by antimalarial drugs. Biochem Pharmacol. 1999;58(1):59-68.

60. Bustos DG, Canfield CJ, Canete-Miguel E, Hutchinson DB. Atovaquoneproguanil compared with chloroquine and chloroquine-sulfadoxinepyrimethamine for treatment of acute Plasmodium falciparum malaria in the Philippines. J Infect Dis. 1999;179(6):1587-90.

61. Hallett RL, Dunyo S, Ord R, Jawara M, Pinder M, Randall A, et al. Chloroquine/sulphadoxine-pyrimethamine for gambian children with malaria: transmission to mosquitoes of multidrug-resistant Plasmodium falciparum. PLoS Clin Trials. 2006;1(3):e15.

62. Menard D, Madji N, Manirakiza A, Djalle D, Koula MR, Talarmin A. Efficacy of chloroquine, amodiaquine, sulfadoxine-pyrimethamine, chloroquinesulfadoxine-pyrimethamine combination, and amodiaquine-sulfadoxinepyrimethamine combination in central African children with noncomplicated malaria. Am J Trop Med Hyg. 2005;72(5):581-5.

63. Vial HJ, Philippot JR, Wallach DFH. A reevaluation of the status of cholesterol in erythrocytes infected by Plasmodium knowlesi and P. Falciparum. Mol Biochem Parasitol. 1984;13(1):53-65.

64. Grellier P, Valentin A, Millerioux V, Schrevel J, Rigomier D. 3-Hydroxy-3methylglutaryl coenzyme a reductase inhibitors lovastatin and simvastatin inhibit in vitro development of Plasmodium falciparum and Babesia divergens in human erythrocytes. Antimicrob Agents Chemother. 1994; 38(5):1144-8.

65. Jomaa H, Wiesner J, Sanderbrand S, Altincicek B, Weidemeyer C, Hintz M, et al. Inhibitors of the nonmevalonate pathway of isoprenoid biosynthesis as antimalarial drugs. Science. 1999;285(5433):1573-6.

66. Ginsburg H. Progress in in silico functional genomics: the malaria metabolic pathways database. Trends Parasitol. 2006;22(6):238-40.

67. Tahar R, Basco LK. Molecular epidemiology of malaria in Cameroon. XXV. In vitro activity of Fosmidomycin and its derivatives against fresh clinical isolates of Plasmodium falciparum and sequence analysis of 1-deoxy-DXylulose 5-phosphate Reductoisomerase. Am J Trop Med Hyg. 2007;77(2): 214-20

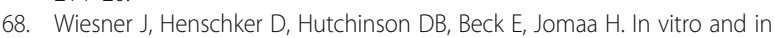
vivo synergy of fosmidomycin, a novel antimalarial drug, with clindamycin. Antimicrob Agents Chemother. 2002;46(9):2889-94.

69. Michael RO, Williams GM. Choloroquine inhibition of repair of DNA damage induced in mammalian cells by methyl methanesulfonate. Mutat Res Mol Mech Mutagen. 1974;25(3):391-6. 